\title{
Vesico-vaginal fistula and bladder stone caused by a protruding spiral tacker 4 years after a laparoscopic sacrocolpopexy: Case report
}

\author{
Gabriella Mirabile ${ }^{1}$, Alfonso Rossetti ${ }^{2}$, Barbara Cristina Gentile ${ }^{1}$, Roberto Giulianelli ${ }^{1}$, \\ Manlio Schettini ${ }^{1}$ \\ ${ }^{1}$ Department of Urology, Nuova Villa Claudia Rome, Italy; \\ 2 Department of Gynecology, Nuova Villa Claudia Rome, Italy.
}

\begin{abstract}
Summary Our case report demonstrates that the use of tackers or other devices in a improper way should be avoid and it can be correlated with late complication of laparoscopic colposacropexy.
\end{abstract}

KEY WORDS: Tacker; Bladder stone; Vesico-vaginal fistula; Sacrocolpopexy.

Submitted 3 September 2015; Accepted 8 December 2015

\section{INTRODUCTION}

We report a case of bladder mesh erosion resulting in a stone and vesico-vaginal fistula due to spiral tacker used for vaginal wall mesh fixation during laparoscopic sacrocolpopexy. To our knowledge, this is the first described case of such a complication.

\section{Case report}

A 67-year-old female presented for an urodynamic study for incontinence. In the 2010 she underwent laparoscopic sacrocolpopexy for vaginal vault prolapse. She complained of urinary symptoms, burning sensation and urgency since 3 years.

A month before severe incontinence appeared, so that she used about 4 pads/day. At ultrasonography a hyperecogenic formation was noticed, attributed to a $4 \mathrm{~cm}$ bladder stone. The urethro-cystoscopy confirmed the presence of the stone attached to the posterior bladder wall at the point of mesh erosion.

We also performed a uretro-cystography (Figure 1) which showed the presence of tackers behind the stone and a vesico-vaginal fistula.

A laparotomy and cystolithotomy was performed and showed a big stone developed on the eroded mesh, which resulted to have been fixed with spiral tackers. The spiral tacker, had eroded into the bladder resulting in a stone and a vesico-vaginal fistula.

The stone was removed with the mesh and all the tackers, and the fistula had been repaired with the interposition of omentum. Fifteen days after surgery, the incontinence due to the fistula persisted, and the catheter was maintained for 15 days more, but cystography confirmed the persistence of the fistula.

A combined vaginal and laparoscopy approach was performed. From the vagina a $12 \mathrm{~F}$ Foley catheter was inserted in the fistula to "mark" it for the laparoscopic approach. Once visualized, the fistula was repaired with a suture, and the water-tightness of the bladder proved. The catheter was removed without complication 30 days after. One year after surgery the patient was asymptomatic and completely dry.

\section{Figure 1.}

Radiography pre cystography.

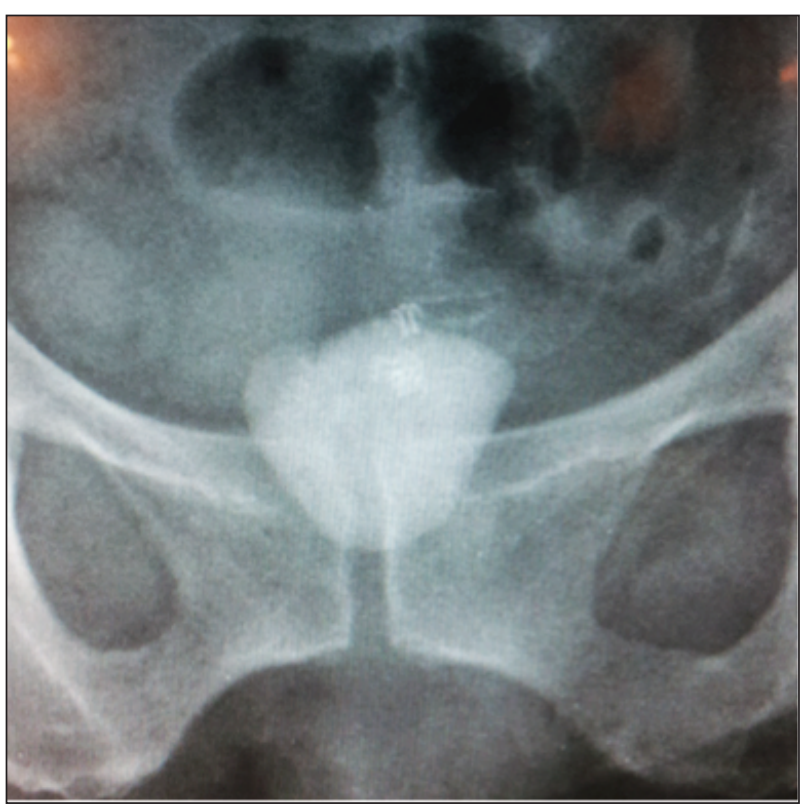

\section{Conclusions}

Mesh fixation with tacker systems is common in laparoscopic inguinal and ventral hernia repair but it is described also for promontofixation. Complications due to tackers are rare.

There are reports of tacker related complications of adhesions, pain, hernia, intestinal obstruction, perforation of 
the bowel or urinary bladder and death. Several cases of spondylodiscitis were reported after use of tackers to the promontory; however this complication also occurs if sutures are utilized. The length of a tacker is $4 \mathrm{~mm}$ long and depending on the thickness of tissue it may penetrate the neighboring structures with disastrous complications.

Alternative techniques of suture fixation of mesh may avoid the tacker related complications.

\section{References}

1. Boukerrou M, Orazi G, Nayama M, et al. Promontofixation procedure: use of non-absorbable sutures or tackers? J Gynecol Obstet Biol Reprod. 2003; 32:524-8.

2. Hazard report. Patient injury or death could result from improper use of U.S. Surgical helical tacks, Health Devices. 2004; 8:293-295.

3. de Tayrac R, Sentilhes L. Complications of pelvic organ prolapse surgery and methods of prevention. Int Urogynecol J. 2013; 24:1859-1872.

\section{Correspondence}

Gabriella Mirabile, MD (Corresponding Author) gabriella.mirabile@urorua.it

Barbara Cristina Gentile, MD

Roberto Giulianelli, MD

Manlio Schettini, MD

Department of Urology, Nuova Villa Claudia via Flaminia nuova 280, Rome, Italy

Alfonso Rossetti, MD

Department of Gynecology, Nuova Villa Claudia via Flaminia nuova 280, Rome, Italy 